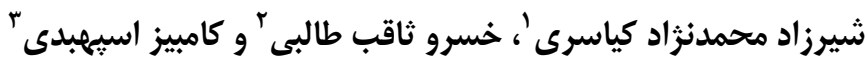

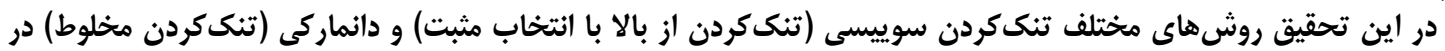

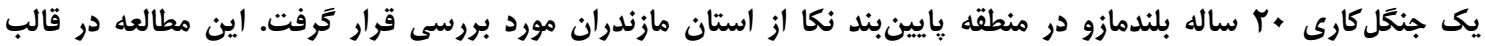

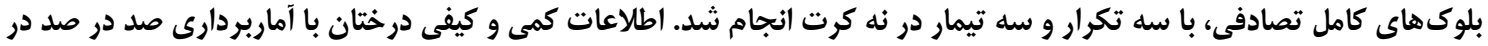

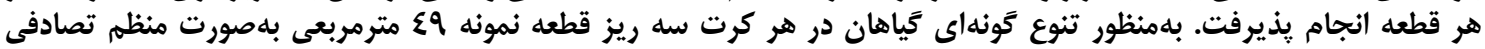

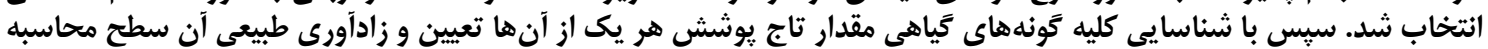

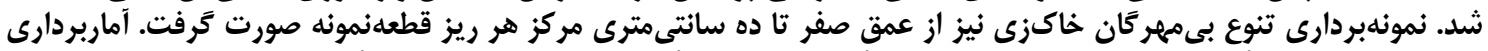

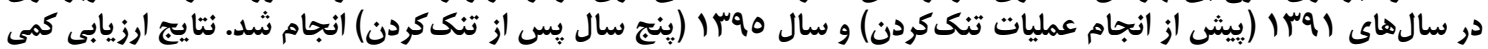

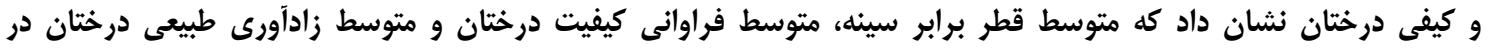

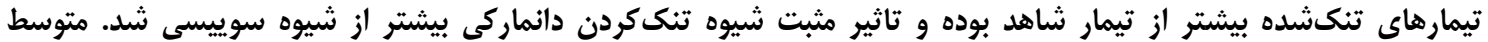

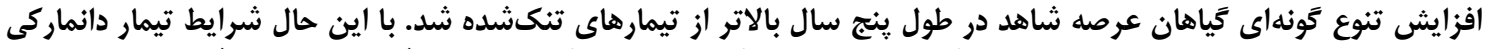

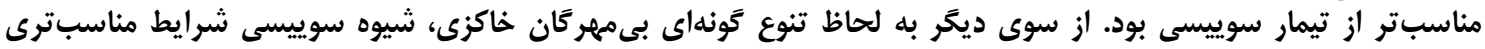

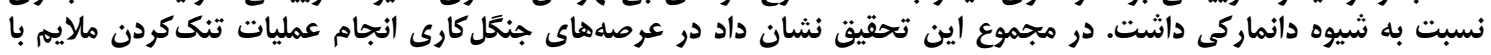
شيوه دانماركى مناسبتر از شيوه سوييسى است.

وازمهاى كليدى: جنكَل كارى، تنككردن، تنوع بىمهركًان خاكزى، تنوع كَونهاى كَياهان، مازندران

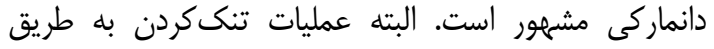

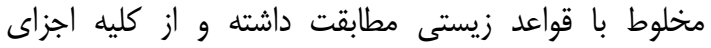

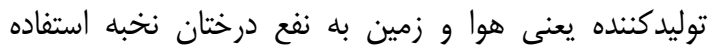

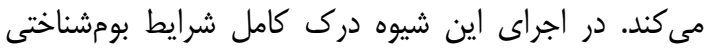

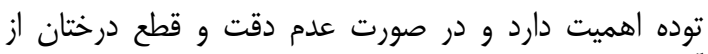

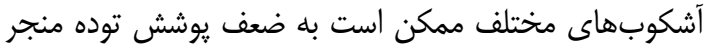

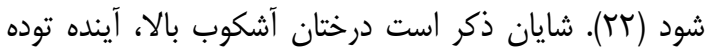

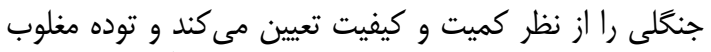

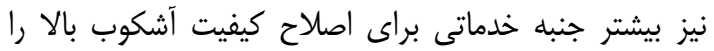

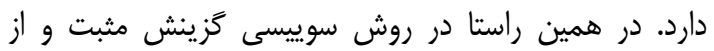

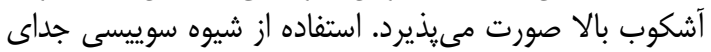

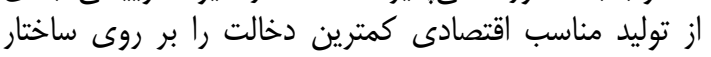

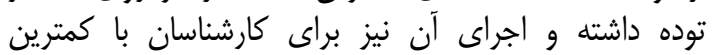

ريسك همراه است (TV،IV) بلندمازو (Quercus castaneifolia C. A. Mey.

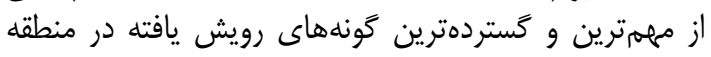

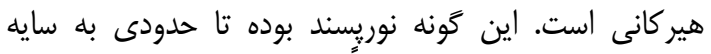

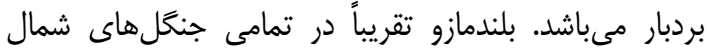

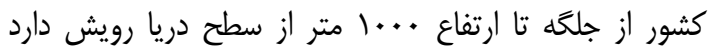

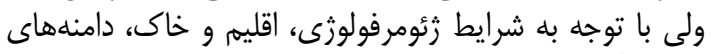

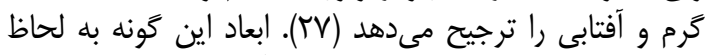

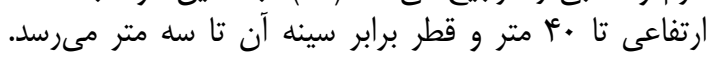

مقدمه عمليات جنغل كارى با بهبود شرايط ريزاقليم (ه)، ايجاد بستر

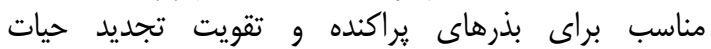

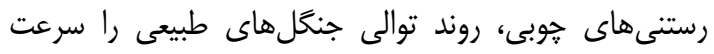

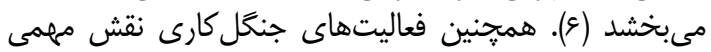

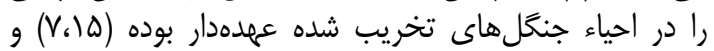

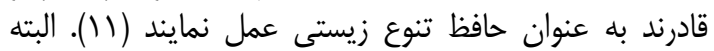

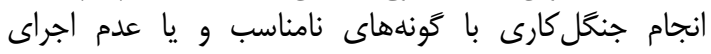

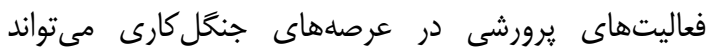

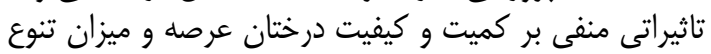

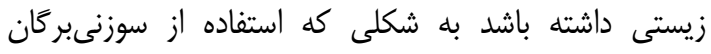

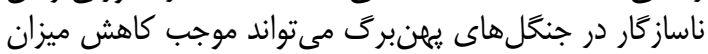

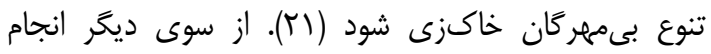

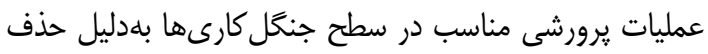

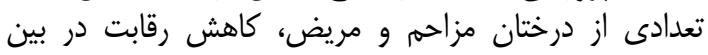

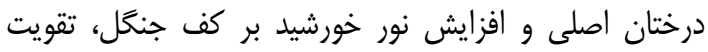

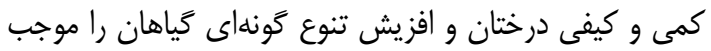
مى شود (r) (r). تنايج تحقيقات مختلف تاثير مثبت انجام عمليات

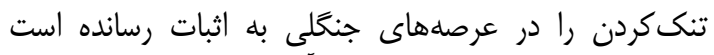

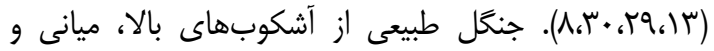

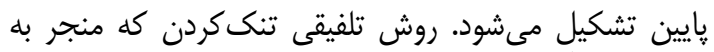

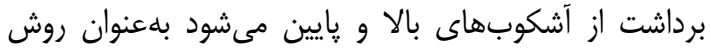


اصلى اين تحقيق نيز آن است كه با استفاده از دو شيوه

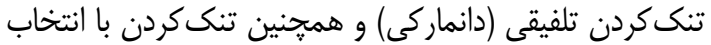

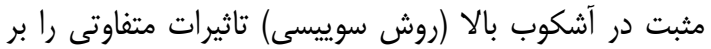

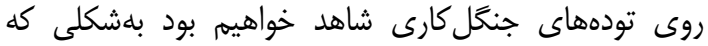

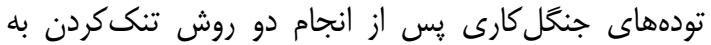

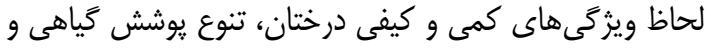

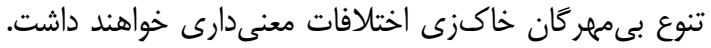

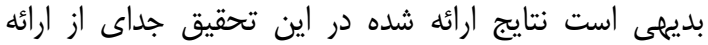

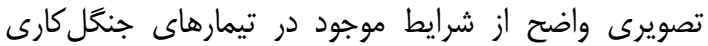

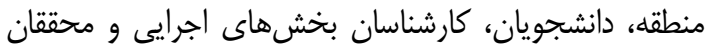

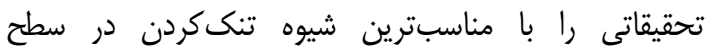
جنكل كارى إىها آشنا مىسازد.

\section{مواد و روشها موردا منطقه مورد مطالعه}

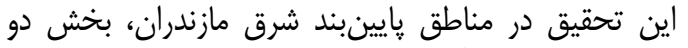

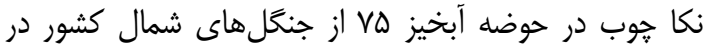

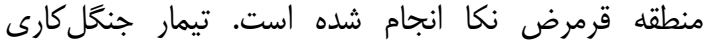

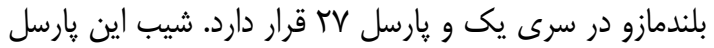

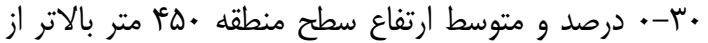

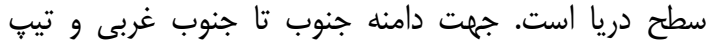

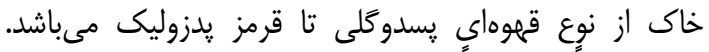

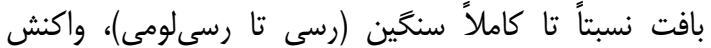

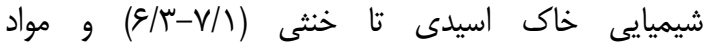

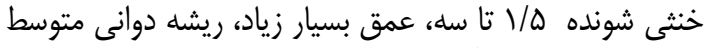
و ميزان نفوذيذيرى آن متوسط تا ضعيف است (شكل ()).
كونه بلندمازو داراى ريشهاى عميق بوده و خواهان خاكهاى شنى آهكى است (1) (1).

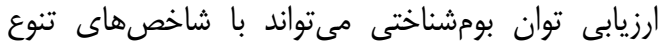

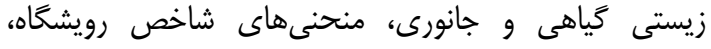

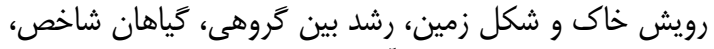
تجزيه برى و لاشبرى

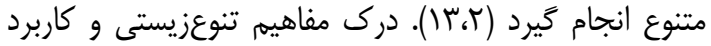

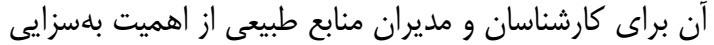

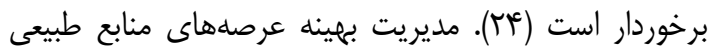

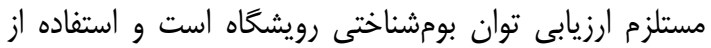

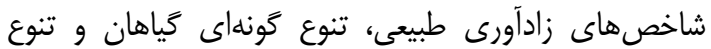

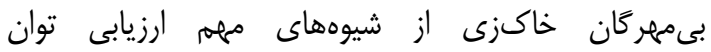

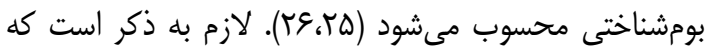

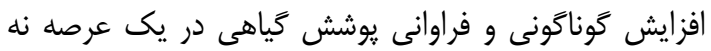

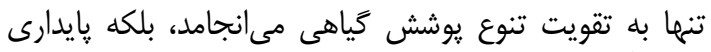

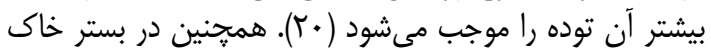

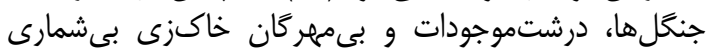

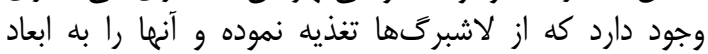

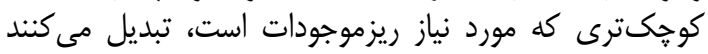

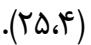

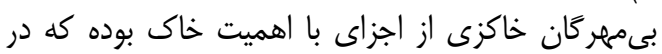

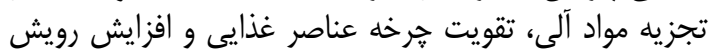

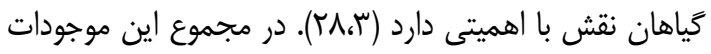

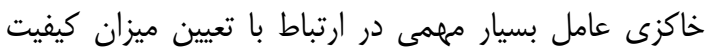

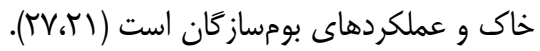

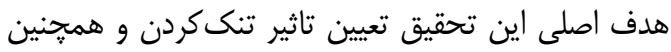

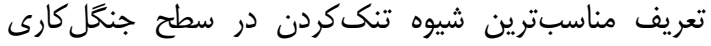
بلندمازو در مناطق پإيينبند استان مازندران است. فرضين

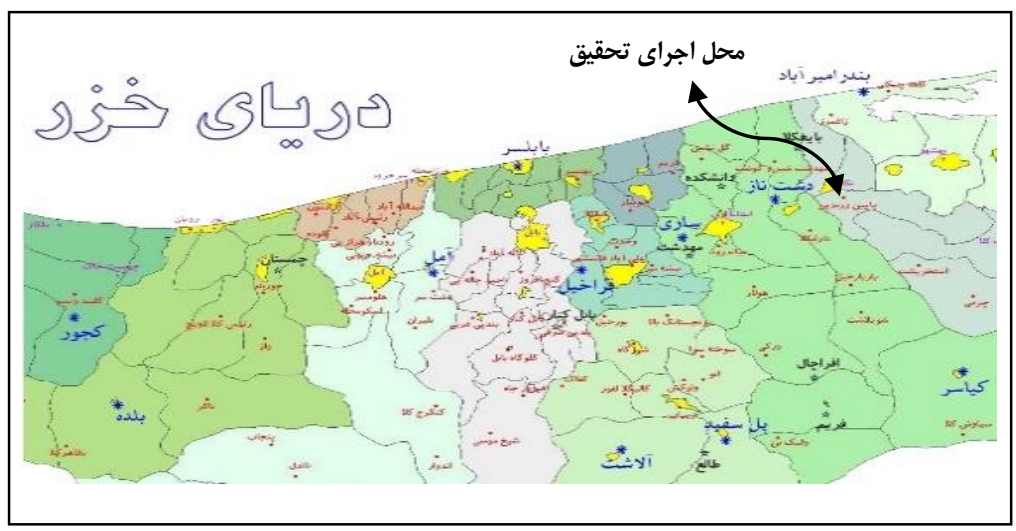

شكل ا- محل اجراى تحقيق در جنگل هاى يايينبند نكا- مازندران

Figure 1. Location of research in the low forest of Neka-Mazandaran

با //سז ميلىمتر داراى كمترين ميزان بارندگى است. با توجيه

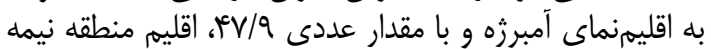

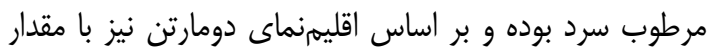

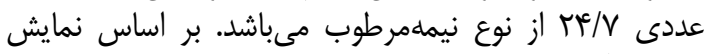

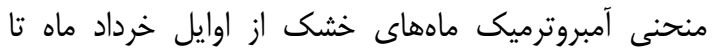
اواسط شهريور ماه است (9).
بر اساس اطلاعات ايستخاه هواشناسى جلمردى (نكا) و در

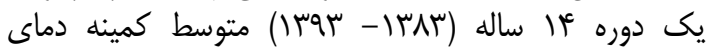

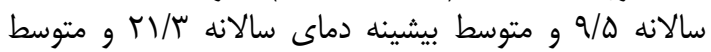

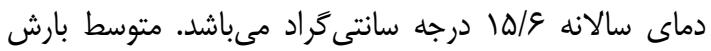

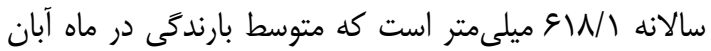
با VD/F ميلىمتر داراى بيشترين ميزان بارندكى و ماه خرداد 
شناسايى كونهها، فراوانى كونه به همراه تاج يوشش هر يك از

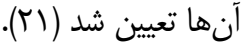

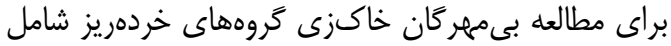

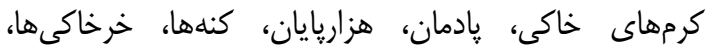

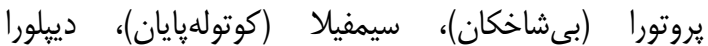

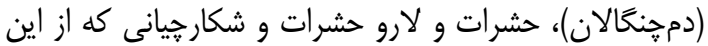

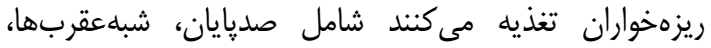

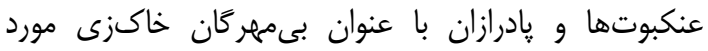

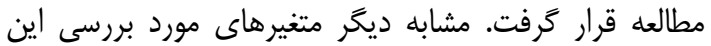

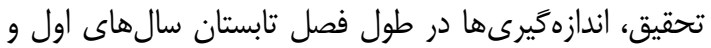

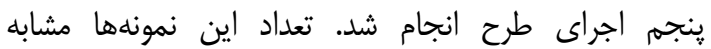

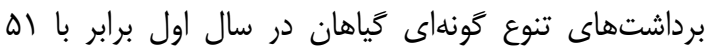

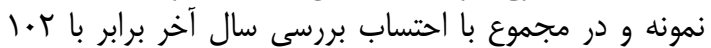

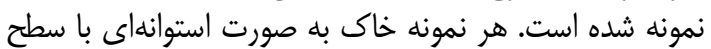

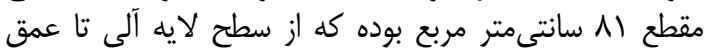

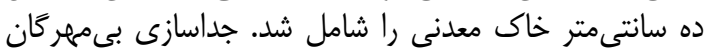

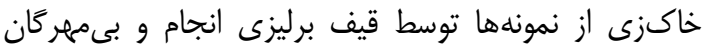

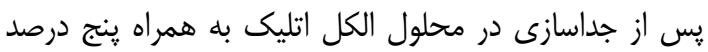

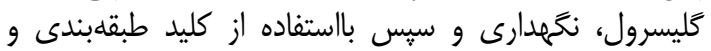

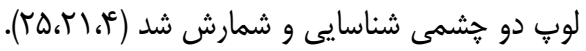

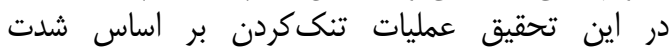

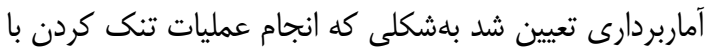

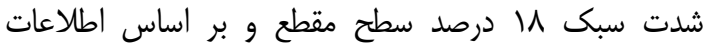

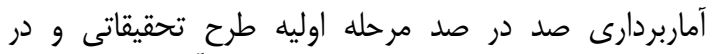

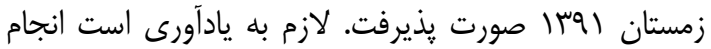

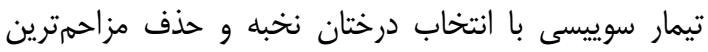

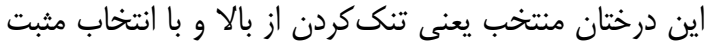

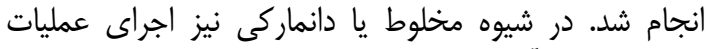

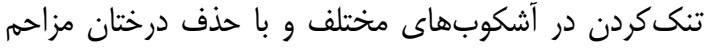

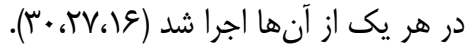

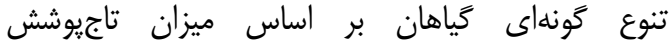

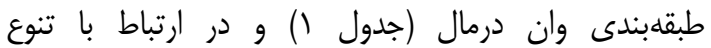

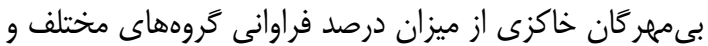

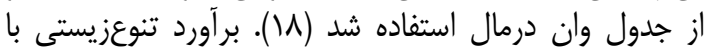

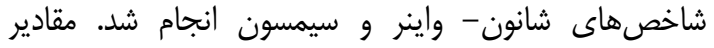

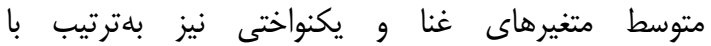

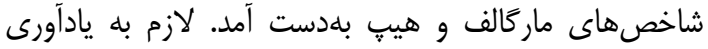

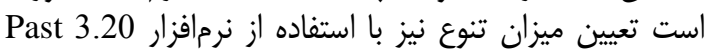

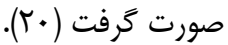

در انجام اين تحقيق با جنكَلَردشى ابتدا نسبت به به

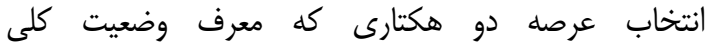

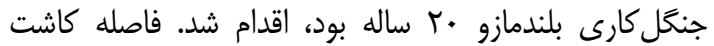

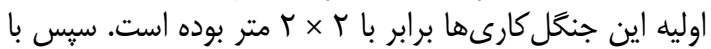

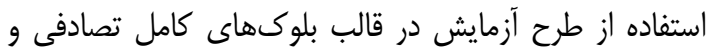

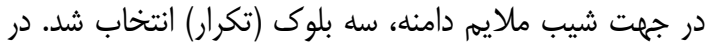

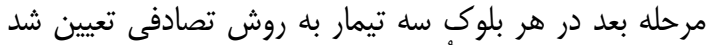

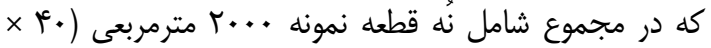

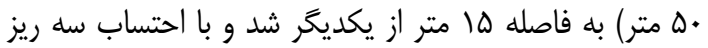

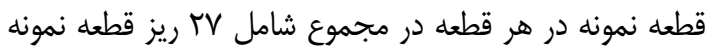

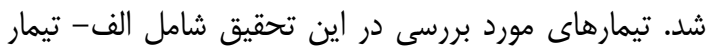

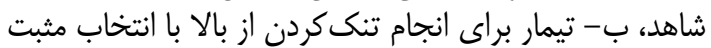

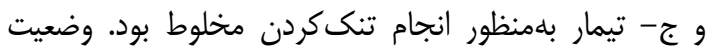

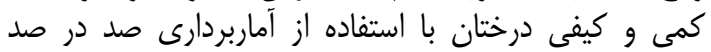

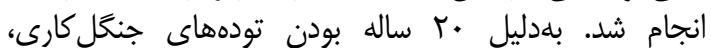

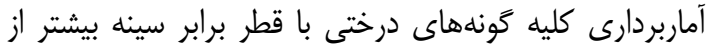

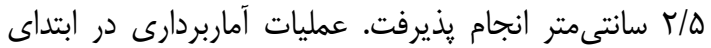

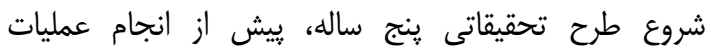

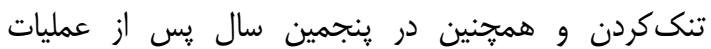

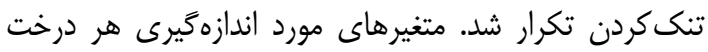

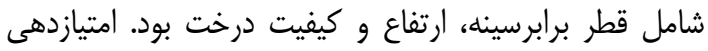

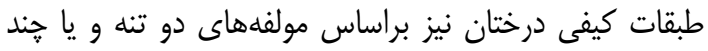

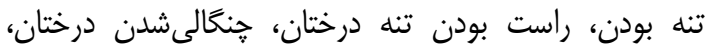

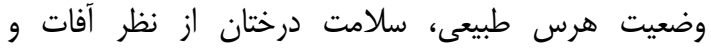

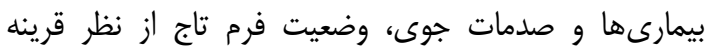

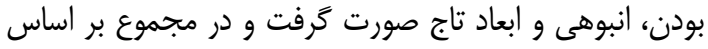

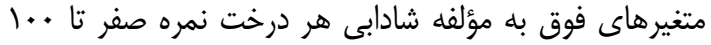
به شرح زير داده شد (بام):

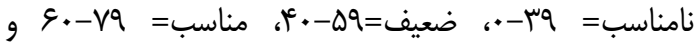

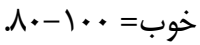
مساحت ريز قطعه نمونههاى بررسى تنوع گَونهاى كياهان

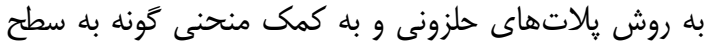

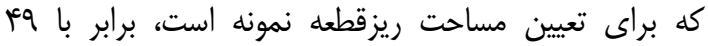

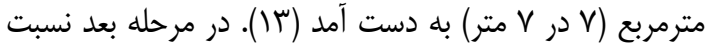

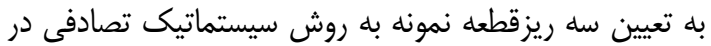

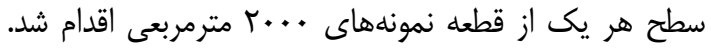
لازم به توضيح است در سطح ريزقطعه نمونهها ضمن 
Table 1. Plant cover according to Wandermal classification

جدول ا- طبقات يوششى براساس طبقهبندى وان درمال

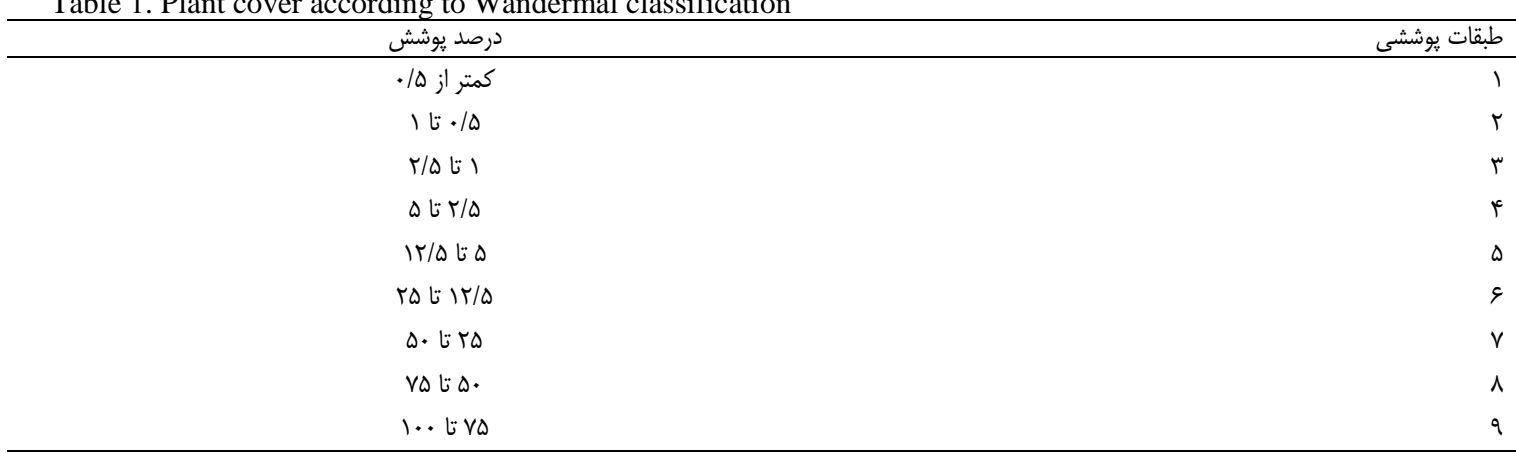

نتايج و بحث بحثيث بررسى وضعيت كمى و كيفى و زادآورى طبيعى

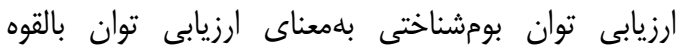

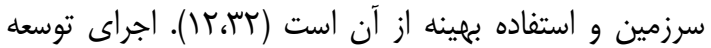

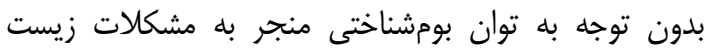

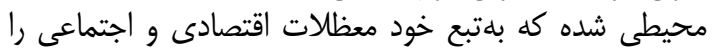

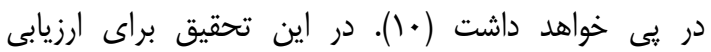

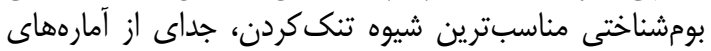

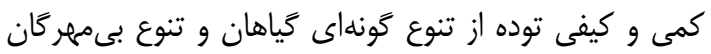
خاكزى استفاده شد.

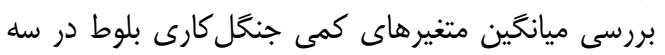
تيمار شاهد، تيمار تنك كردن تلفيقى (روش دانئ دانماركى) و وتيمار

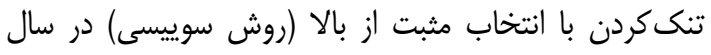

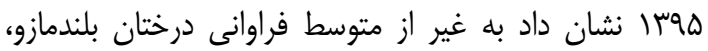

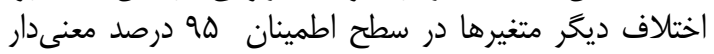

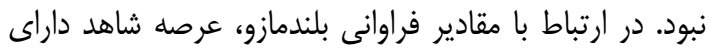

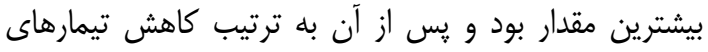
دانماركى و سوييسى قرار كرفت (جدول كار آن.
تجزيه و تحليل ميانكَين أمارههاى كمى در اين تحقيق با بادي

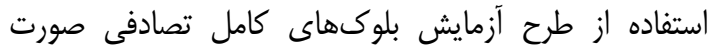

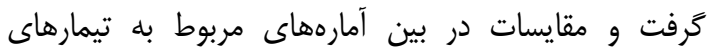

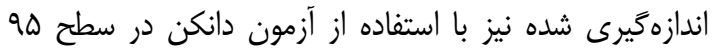

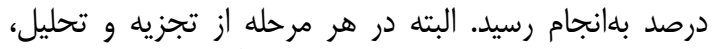

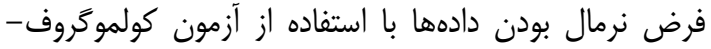

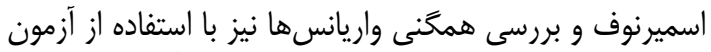

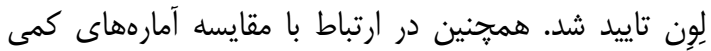

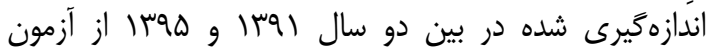
تى تست مستقل استفاده شد. تجزيه و تحليل كيفى درختان نيز إنقاد در دو در مرحله انجام

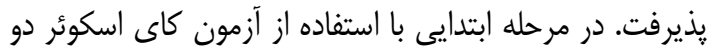

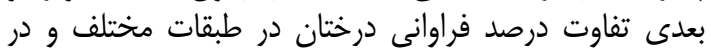

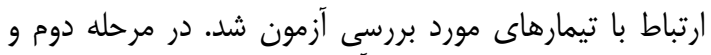

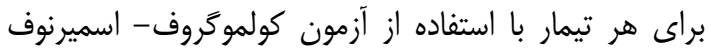

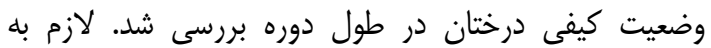

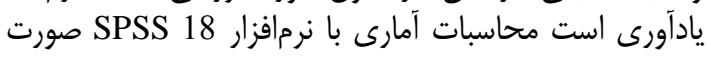

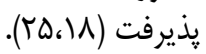

جدول r- مشخصات كمى درختان در سطح جنگَل كارى بلندمازو با دو تيٍ تنكى كردن درسال هوسן Table 2. Quantitative assessment of trees and natural regeneration in the Oak plantation, 2017

\begin{tabular}{|c|c|c|c|}
\hline \multicolumn{3}{|c|}{ روش تنك كردن } & \multirow[b]{2}{*}{ متغيرهاى مورد بررسى } \\
\hline انتخاب مثبت از بالا (سوييسى) & انتخاب از بالا و پايين (دانماركى) & (بدون دخالت) & \\
\hline VפT/KY (IVT/NF $)^{\mathrm{ns}}$ & $M \Delta(r / r / \Lambda F)^{n S}$ & $11 \cdots(190)^{\mathrm{ns}}$ & فراوانى درخت در هكتار \\
\hline$C \cdot \Delta(V / / q \Delta)^{b}$ & $r \mu / g V(\mu N / \Lambda r)^{b}$ & $\varepsilon \Lambda \mu / \mu r(\Lambda \cdot / \mu q)^{\mathrm{a}}$ & فراوانى كَونه اصلى در هكتار \\
\hline $10 / V^{E}(\cdot / 9 \cdot)^{\mathrm{ns}}$ & $\mid r / \cdot V(V / \Delta r)^{n s}$ & $\mathbb{I r / \Lambda \mu}(\cdot / \Lambda \mathrm{V})^{\mathrm{ns}}$ & قطر برابرسينه توده (سانتىمتر) \\
\hline $\operatorname{IV/va}(r / v q)^{\mathrm{ns}}$ & 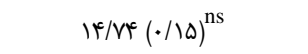 & 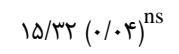 & قطر برابرسينه كَونه اصلى (سانتىمتر) \\
\hline $\mid r / M(\cdot / F \Delta)^{\mathrm{ns}}$ & $|r / F|(\cdot / \kappa r)^{\mathrm{nS}}$ & $11 / v \varepsilon(\cdot / 19)^{\mathrm{ns}}$ & متوسط ارتفاع توده جنگكل كارى (متر) \\
\hline $\mid r /{ }^{\prime} \Lambda(\cdot / r \cdot)^{\mathrm{ns}}$ & $\mid r / r r(\cdot / \cdot c)^{\text {ns }}$ & $\mid r / 99(\cdot / \cdot \Delta)^{\mathrm{ns}}$ & متوسط ارتفاع كَونه اصلى (متر) \\
\hline V৭/9r $(19 / 98)^{\mathrm{ns}}$ & $V \varepsilon / T r(r g / I T)^{\mathrm{nS}}$ & 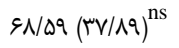 & فراوانى زادآورى در ريزقطعلنمونه (اصله) \\
\hline
\end{tabular}

(كاهش FV

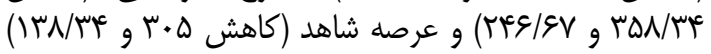

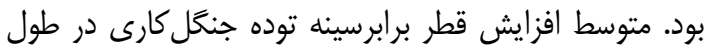
ينج سال بهترتيب كاهش شامل شيوه دانماركى (افزايش
در مرحله بعد تفاضل متوسط مقادير هر يك از متغيرهاى

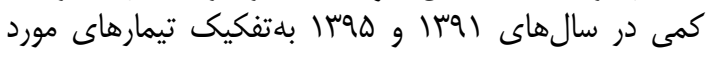

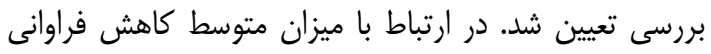

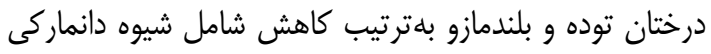


موجب افزايش آمارههاى متوسط قطر برابرسينه توده و

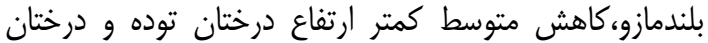

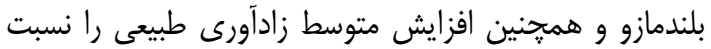

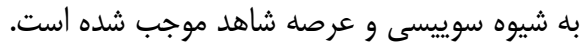

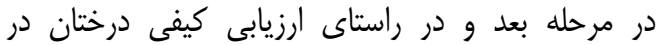

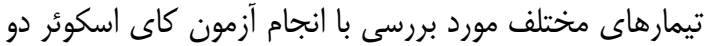

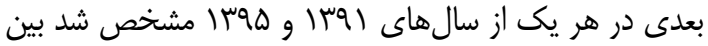

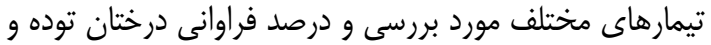

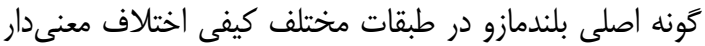

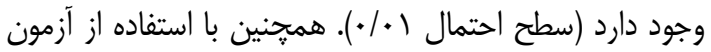

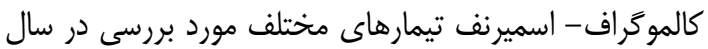

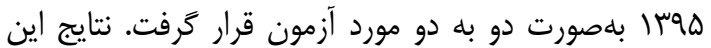

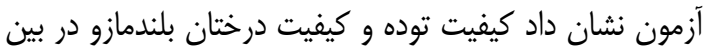

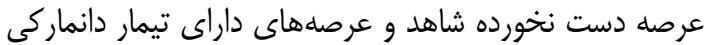

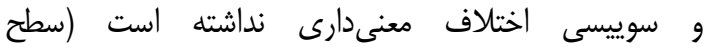

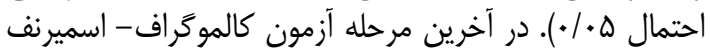

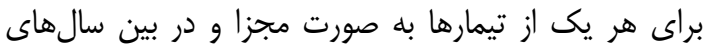

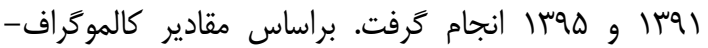

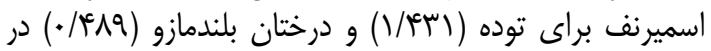

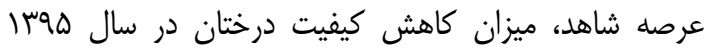

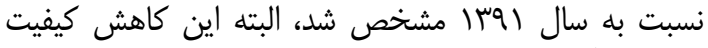

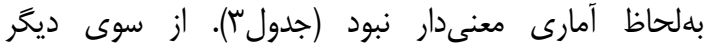

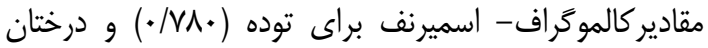

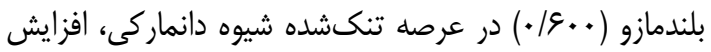

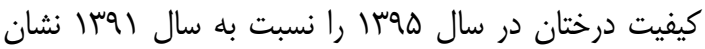

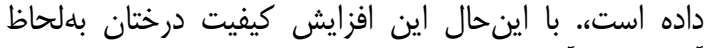

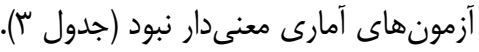

وس/ سانتىمتر)، عرصه شاهد (افزايش ع سץ/ץ سانتىمتر) و

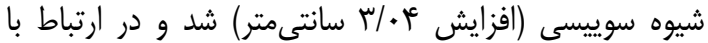

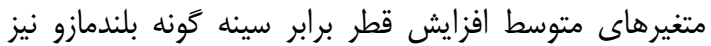

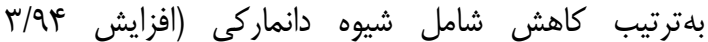

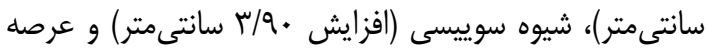

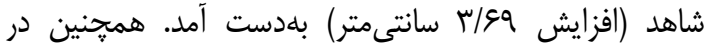

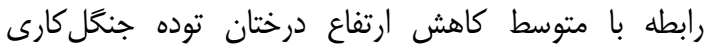

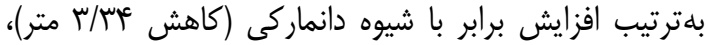

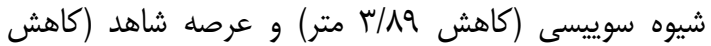

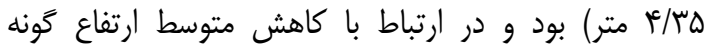

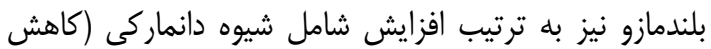

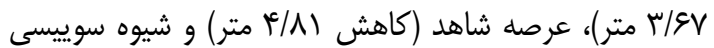

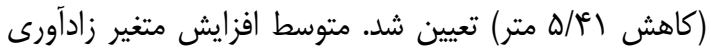

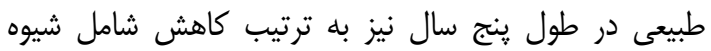

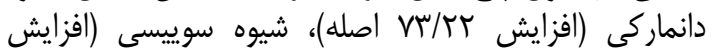

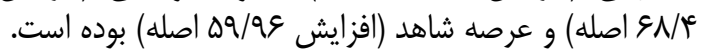
در تحقيق حاضر شدت بردات برداشت شيوهنهاى مختلف

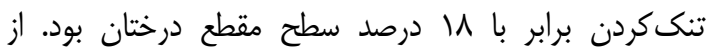

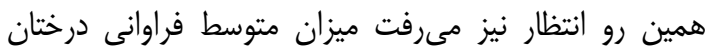

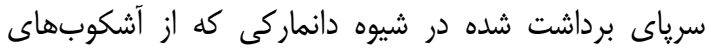

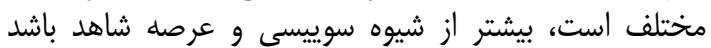

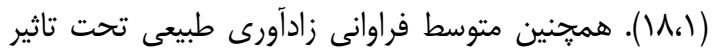

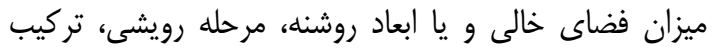

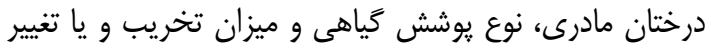

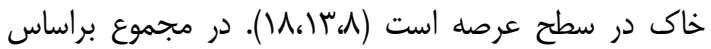

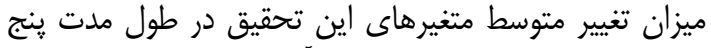
سال اجراى طرح كه در يرانتزها آمده است، شيوه دئ دانماركى ملى مدي

جدول س- درصد فراوانى كيفى درختان براى درجات مختلف كيفيت در عرصه جنكَل كارى بلندمازو Table 3. The percent of frequency of trees in different quality classes in the Oak plantation

\begin{tabular}{|c|c|c|c|c|c|c|c|}
\hline \multicolumn{2}{|c|}{ (روش سوييسى) انتخاب مثبت ازلا } & \multicolumn{2}{|c|}{ انتخاب از بالا و يايين } & \multicolumn{2}{|c|}{ (بدون تنككردن) } & \multicolumn{2}{|c|}{ درجات كيفيت } \\
\hline$|r q|$ & 1490 & $\mid r q 1$ & $1 \% 90$ & 1491 & 1490 & & \\
\hline re/AD & $r \Delta / \Lambda)$ & שT & $r \Delta / \mathcal{N Q}$ & $r V / \kappa q$ & $r F / . q$ & وضعيت خوب & \\
\hline$r \cdot / \Lambda$. & $19 / r 1$ & $r / \Delta q$ & tr & שו/אז & $r r / v q$ & وضعيت مناسب & $3:$ \\
\hline$\Gamma F / \Lambda)$ & $r V / r q$ & rN/q9 & $r \Delta / 8 q$ & $r r / \wedge 9$ & $19 / \wedge \Delta$ & وضعيت ضعيف & 5 \\
\hline$I V / D F$ & $\mid V / 9 q$ & $14 / 99$ & $18 / 50$ & $18 / 19 q$ & $T r / T V$ & وضعيت نامناسب & \\
\hline$F N / D Q$ & $\Delta \cdot / T)$ & rq/ & $F T / V \Delta$ & $r \Delta / 4 \varphi$ & $F r / q 4$ & وضعيت خوب & \\
\hline$r \cdot / V T$ & 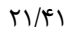 & $r \cdot / 91$ & $r .191$ & $r / \Delta \mu$ & r & وضعيت مناسب & 3 \\
\hline $19 / 99$ & $|N / 1|$ & $r r / v \Lambda$ & 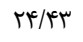 & $\leftarrow \varepsilon / 11$ & $r$. & وضعيت ضعيف & 恿 \\
\hline 11 & $1 . / T \Lambda$ & $\mid Q / A D$ & $|r / r|$ & $\mid r / \Delta \Lambda$ & $|r / 4|$ & وضعيت نامناسب & $\alpha$ \\
\hline
\end{tabular}

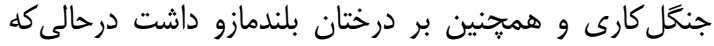

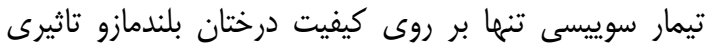

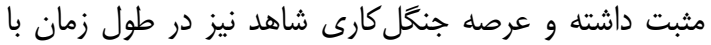

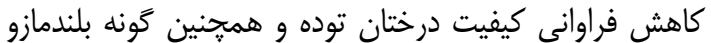

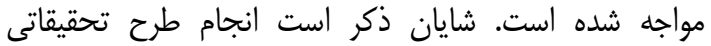

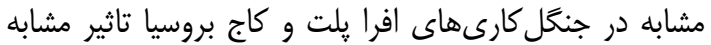

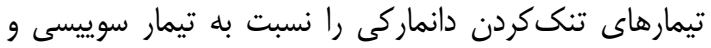
عرصه شاهد نشان داده است (1) تئمان دانماركي

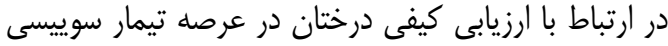

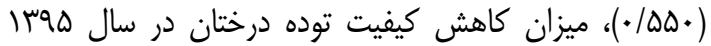

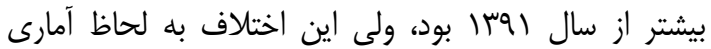

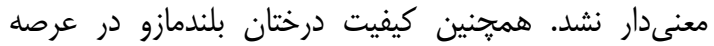

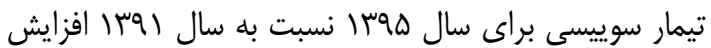

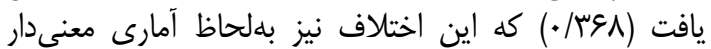

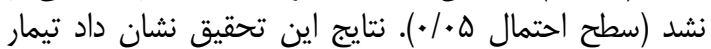

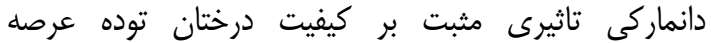




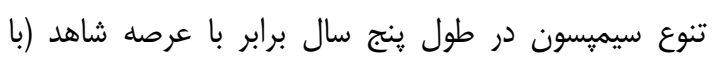

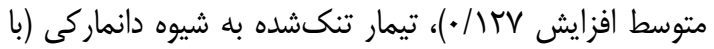

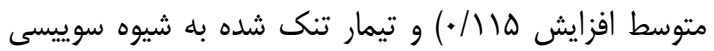
(با متوسط افزايش و9.

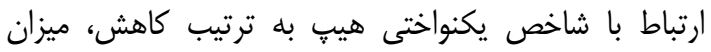

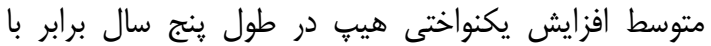

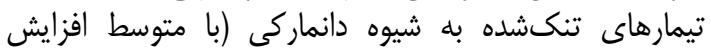

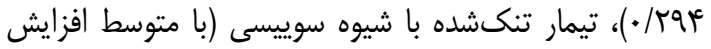

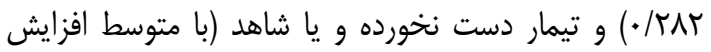

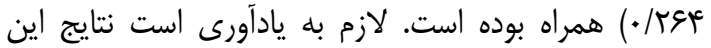

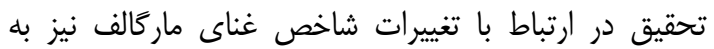

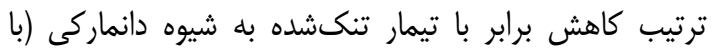

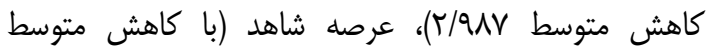

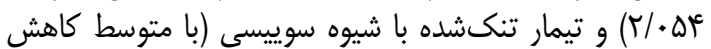

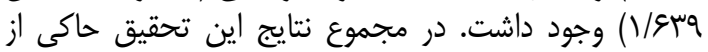

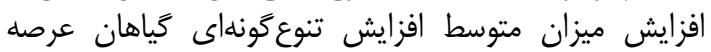

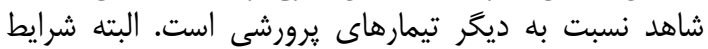
تيمار دانماركى مناسبتر از تيمار سوييسى بوده است (جدول

انجام تحقيقى مشابه در سطح جنكل كارى رِّلت استان

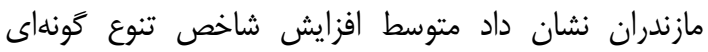

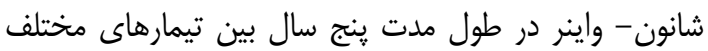

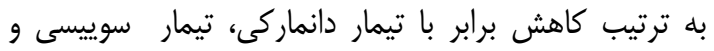
عرصه شاهد بود ("آ).

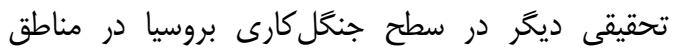

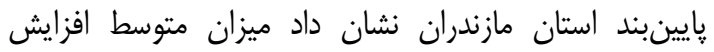

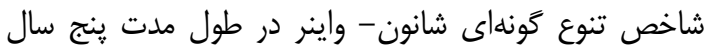

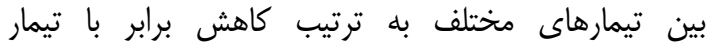

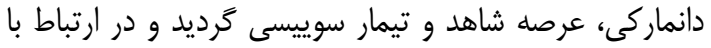

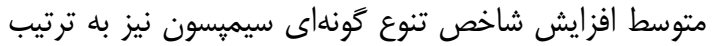

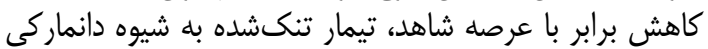

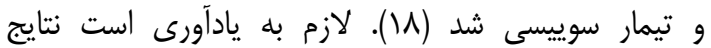

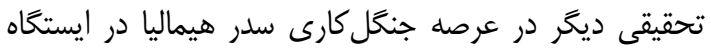

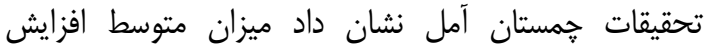

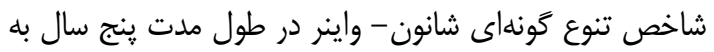

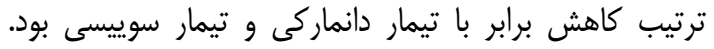

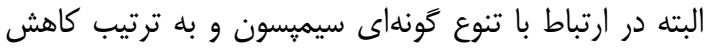

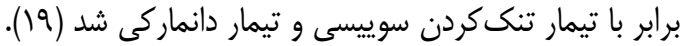

\section{بررسى وضعيت تنوع كَونهاى تَياهان}

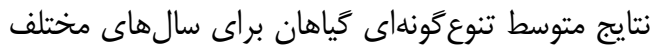

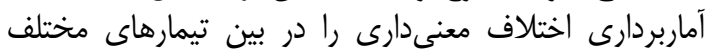

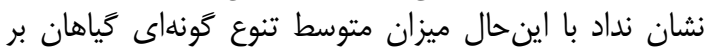

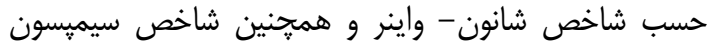

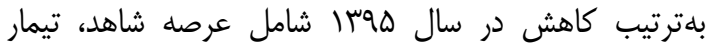

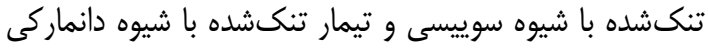

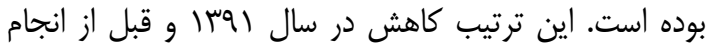

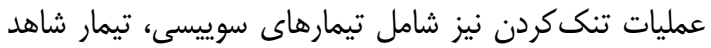

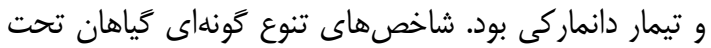

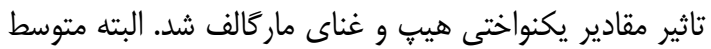

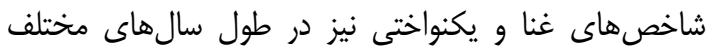

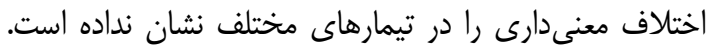

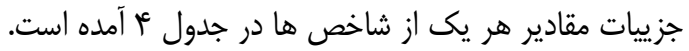

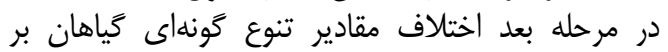

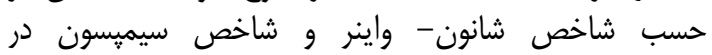

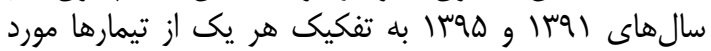

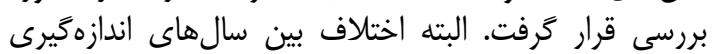

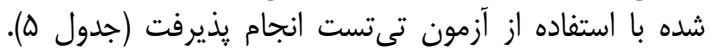

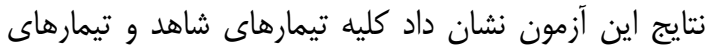

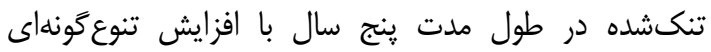

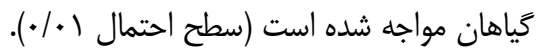

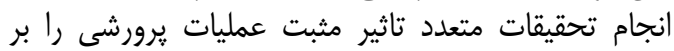

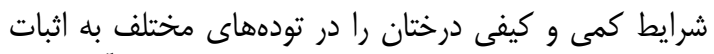

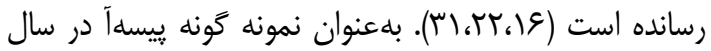

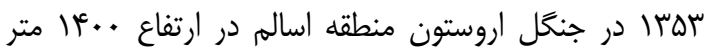

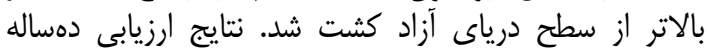

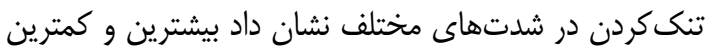

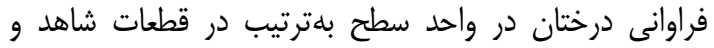

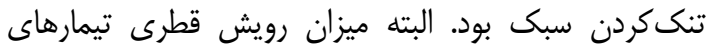

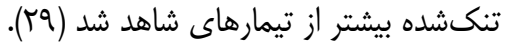

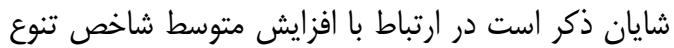

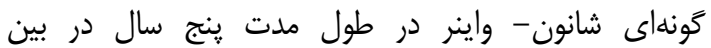

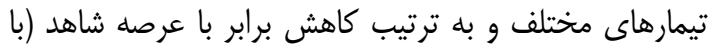

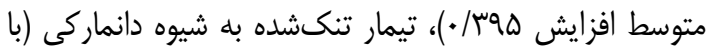

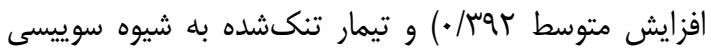

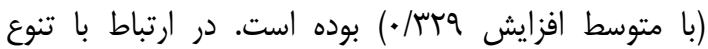

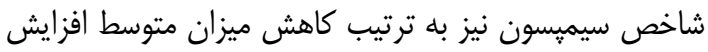


$\xi$. ارزيابى دو شيوه تنك كردن در جنگل كارى بلندمازو بر اساس تعيين بومشناختى

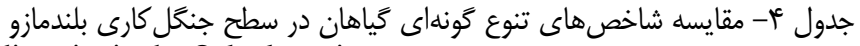
Table 4. The indices comparison of plant species diversity in the Oak plantation

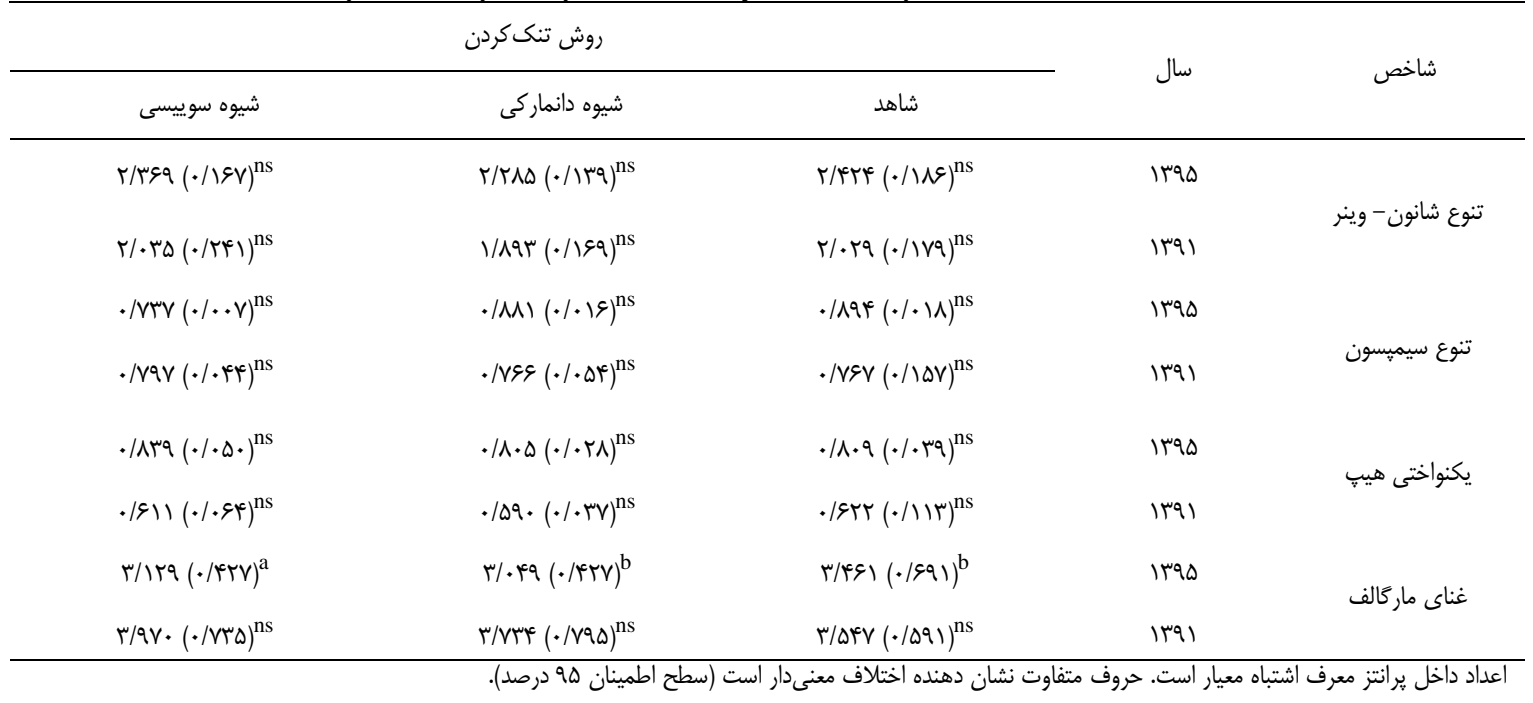

جدول ه- مقايسه شاخصهاى تنوع گَنهاى گياهان در طول سالهاى اqجا و هوسا Table 5. The indices comparison of plant species diversity during the years 2013 and 2017

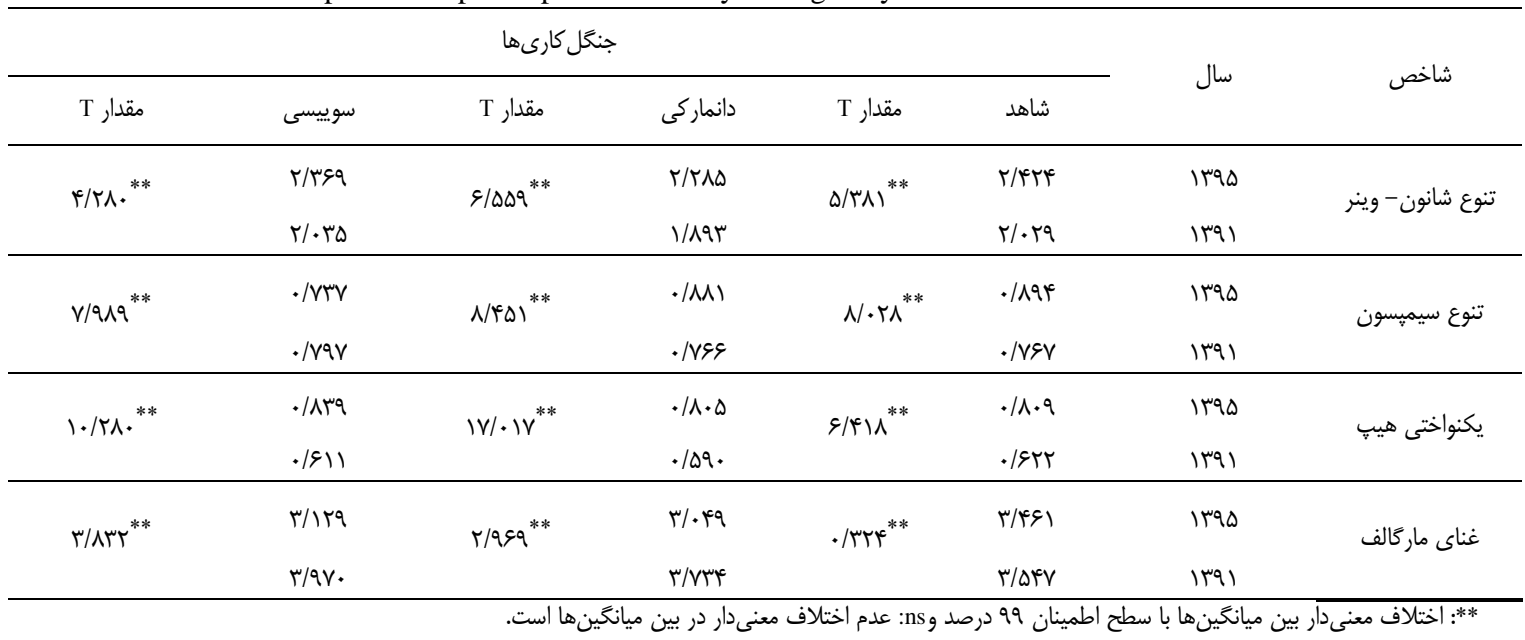

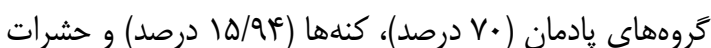

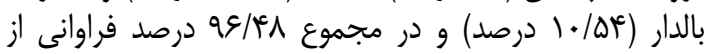

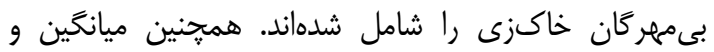

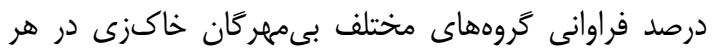

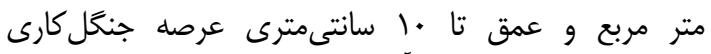
بلندمازو به شرح جدول ع و آمده است.

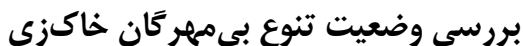

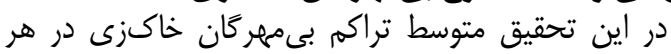

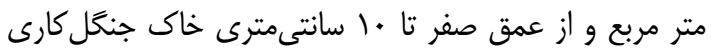

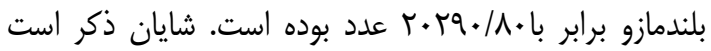

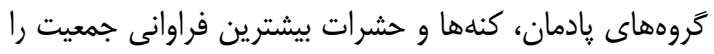

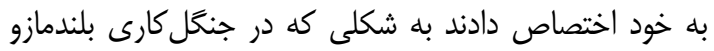


Table 6. Specifications of soil invertebrates per square meter

جدول צ- مشخصات بىمهر گان خاكزى در هر مترمربع

\begin{tabular}{|c|c|c|}
\hline درصد & فراوانى & كروهها \\
\hline $10 / Q \varphi^{c}$ & TrM/DS & كنdهl \\
\hline שe & rAV/qI & كرمهاى خاكى \\
\hline$\cdot / \Delta F$ & $1.9 / 81$ & هزاريايان \\
\hline$\cdot / r$ & FI/Ir & صديايان \\
\hline.$|4|$ & NT/TG & يرويداها \\
\hline$\cdot / T V$ & $\Delta F / \Lambda F$ & خرخاكى ها \\
\hline r/l. & TV/ET & يروتوراها \\
\hline rו/. & TV/et & شبه عقربها \\
\hline $1 \ldots$ & $r \cdot r q \cdot / \Lambda$. & مجموع \\
\hline
\end{tabular}

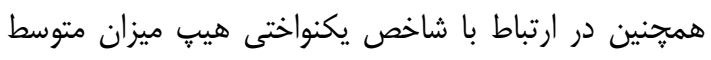

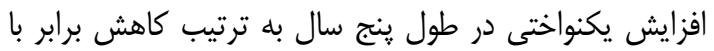

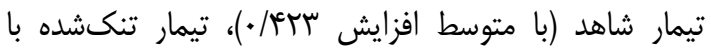

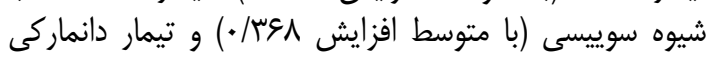

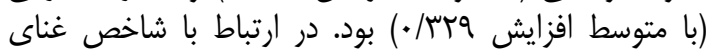

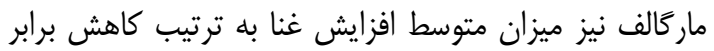

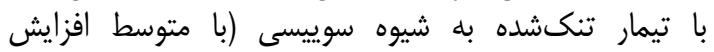

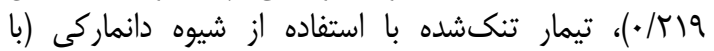

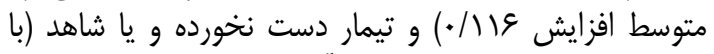

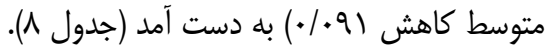

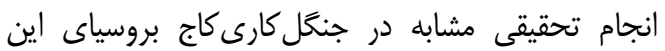

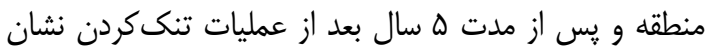

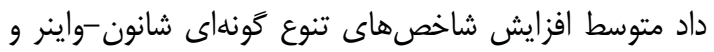

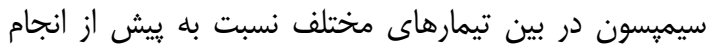

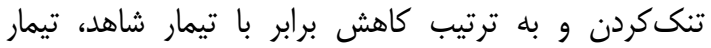

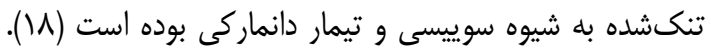

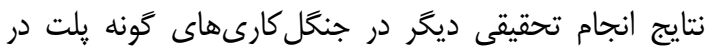

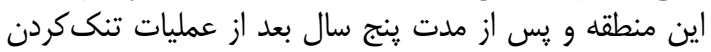

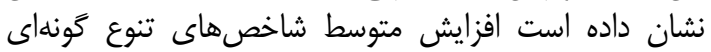

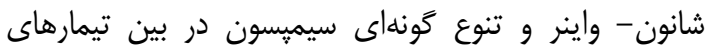

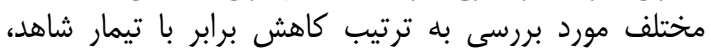

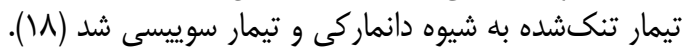
نتايج اين تحقيق در طول ينج

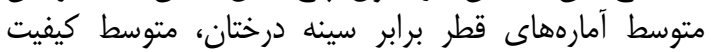

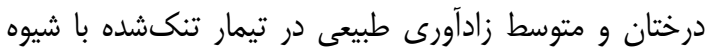

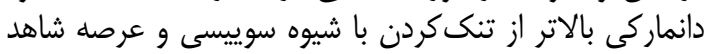

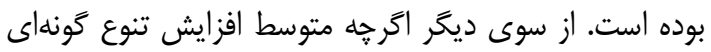

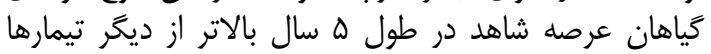

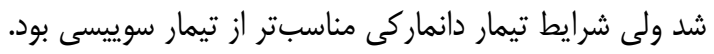

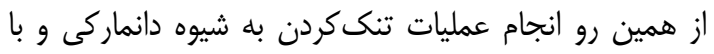

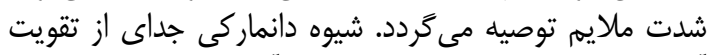

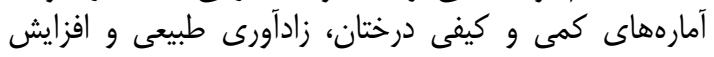

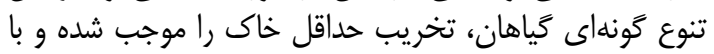

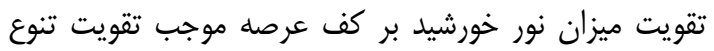
بى مهركان خاكزى شيز نده است.

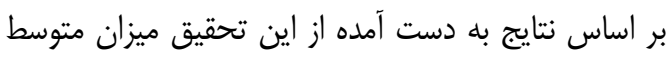

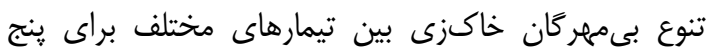

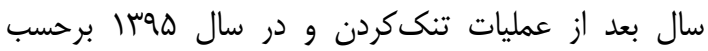

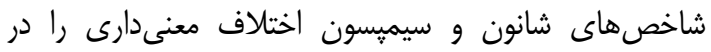

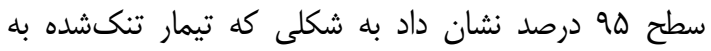

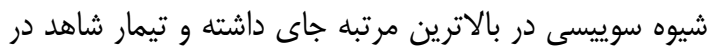

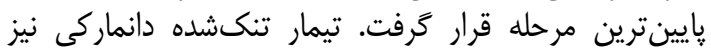

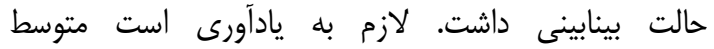

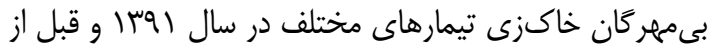

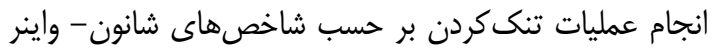

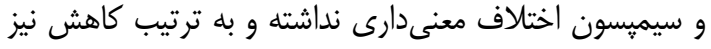

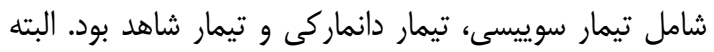

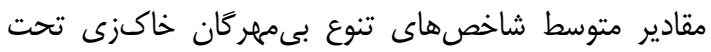

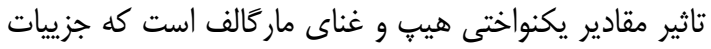

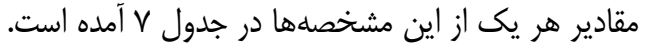

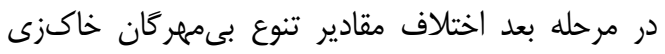

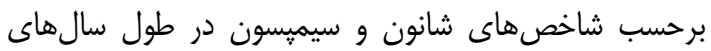

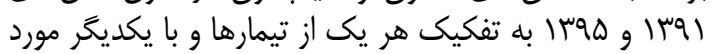

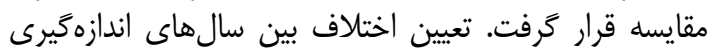

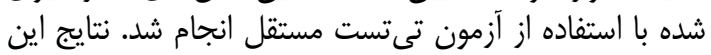

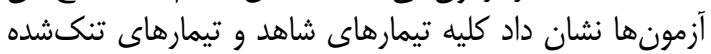

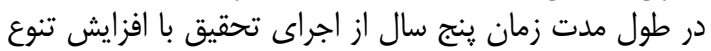

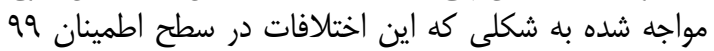

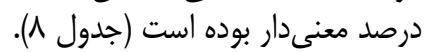

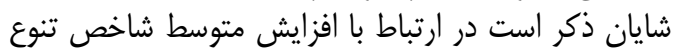

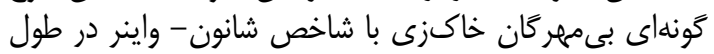

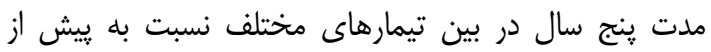

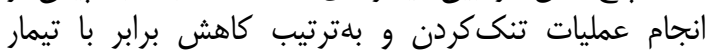

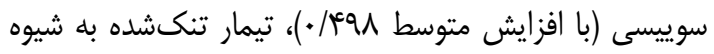

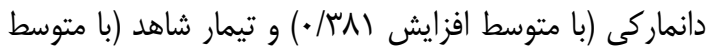

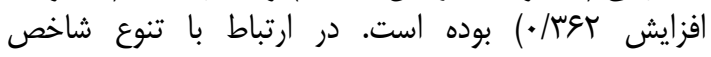

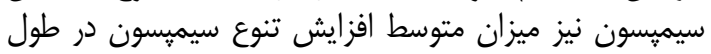

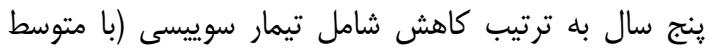

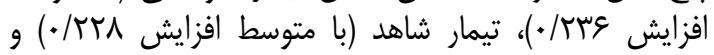

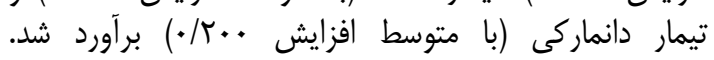




\begin{tabular}{|c|c|c|c|c|}
\hline \multicolumn{3}{|c|}{ روش تنك كردن } & \multirow{2}{*}{ سال س } & \multirow{2}{*}{ شاخص } \\
\hline شيوه سوييسى & شيوه دانماركى & شاهد & & \\
\hline $1 /$ FVq $(\cdot / r V I)^{a}$ & $1 / T \Lambda \backslash(\cdot / \Lambda \Lambda \varepsilon)^{a b}$ & $1 / T T V(\cdot / / F r)^{b}$ & (1790 & \multirow{2}{*}{ تنوع شانون- وينر } \\
\hline$\cdot / 911(\cdot / 194)^{\mathrm{ns}}$ & $\cdot / 9 \cdots(\cdot / 199)^{\mathrm{ns}}$ & $\cdot / N \& \Delta\left(\cdot / r \varphi^{\prime}\right)^{\mathrm{nS}}$ & $|r q|$ & \\
\hline.$/ \mathrm{VQT}(\cdot / .9 \mathrm{~V})^{\mathrm{a}}$ & $\cdot / V \cdot r(\cdot / \cdot \Delta I)^{a b}$ &.$/ 99 \Delta(\cdot / .4 \mu)^{b}$ & (1690 & \multirow{2}{*}{ تنوع سيمِسون } \\
\hline$\cdot / \Delta \mathrm{IV}(\cdot / . q \mu)^{\mathrm{ns}}$ & $\cdot / \Delta \cdot r(\cdot / 1 \cdot r)^{\mathrm{ns}}$ & $\cdot / \kappa \& V(\cdot / l f \varphi)^{\mathrm{ns}}$ & $|r q|$ & \\
\hline$\cdot / 901(\cdot / \cdot r V)^{\mathrm{ns}}$ &.$/ q \uparrow \mathrm{r}(\cdot / \cdot \mathrm{rV})^{\mathrm{ns}}$ & $\cdot / 9 \& 9(\cdot / \cdot r V)^{\mathrm{ns}}$ & (1400 & \multirow{2}{*}{ يكنواختى هيب } \\
\hline$\cdot / \Delta \Lambda \mu(\cdot / T r)^{\mathrm{ns}}$ & $\cdot|q| f(\cdot / 1 \Delta V)^{\mathrm{ns}}$ & $\cdot \mid \Delta+\varepsilon(\cdot /|\Delta|)^{\text {ns }}$ & $|r q|$ & \\
\hline $1 /|\varepsilon|(\cdot / r \omega \cdot)^{\mathrm{ns}}$ & $\cdot / 9 T r(\cdot / \pi r \varepsilon)^{\mathrm{ns}}$ & $\cdot / \operatorname{ArV}(\cdot / / \uparrow q)^{\mathrm{ns}}$ & 1490 & \multirow{2}{*}{ غناى ماركالف } \\
\hline$\cdot / 9 F r(\cdot / r) \cdot)^{\mathrm{ns}}$ & $\cdot|A| \varepsilon(\cdot / K F Y)^{\mathrm{ns}}$ &.$/ 91 \wedge(\cdot / r \mid r)^{\mathrm{ns}}$ & $|r q|$ & \\
\hline
\end{tabular}

جدول ^- مقايسه شاخصهاى تنوع بىمهر گان خاكزى در طول سالهاى اوسا و هوسا Table 8. The indices comparison of soil invertebrates' diversityduring the years 2013 and 2017

\begin{tabular}{|c|c|c|c|c|c|c|c|}
\hline \multicolumn{6}{|c|}{ جنجً كارى ها } & \multirow{2}{*}{ سال } & \multirow{2}{*}{ شاخص } \\
\hline مقدار T & سوييسى & مقدار T T & دانماركى & مقدار T & شاهد & & \\
\hline \multirow[t]{2}{*}{$\Delta / \cdot r f^{* *}$} & $1 / 4 v q$ & \multirow[t]{2}{*}{$\Delta /$ q. $^{* *}$} & $1 / T \Lambda 1$ & \multirow[t]{2}{*}{ r/ałs ${ }^{* *}$} & & & \multirow[t]{2}{*}{ تنوع شانون - وينر } \\
\hline &.$/ 911$ & & $. / 9 .$. & &.$/ N \& \Delta$ & 1491 & \\
\hline \multirow[t]{2}{*}{$V /\left.\& \Lambda\right|^{* *}$} & . /VAT & \multirow[t]{2}{*}{$\left.\operatorname{s|Av}\right|^{* *}$} & $\cdot / v \cdot r$ & \multirow[t]{2}{*}{ s/rা } &.$/ 990$ & $1 \% 90$ & \multirow[t]{2}{*}{ تنوع سيميسون } \\
\hline &.$/ \Delta I V$ & & $.10 . r$ & &.$/ 49 \mathrm{~V}$ & 1491 & \\
\hline \multirow[t]{2}{*}{$I T / T A f^{* *}$} &.$/ 901$ & \multirow[t]{2}{*}{$N / g \mid V^{* *}$} & 4 & \multirow[t]{2}{*}{$11 / \mathrm{v} \wedge^{* *}$} &.$/ 989$ & $1 \% 90$ & \multirow[t]{2}{*}{ يكنواختى هيب } \\
\hline &.$/ \Delta \Lambda \mu$ & &.$\left.|8|\right|^{4}$ & & . /DFG & 1491 & \\
\hline \multirow[t]{2}{*}{$1 / v 98^{\mathrm{ns}}$} & $|/| 9 \mid$ & \multirow[t]{2}{*}{$1 / \pi \times \varepsilon^{\mathrm{ns}}$} & r ז & \multirow[t]{2}{*}{$1 / * 0^{\mathrm{ns}}$} & . /ATV & $1 \% 90$ & \multirow[t]{2}{*}{ غناى ماركالف } \\
\hline & . /945 & & $. / 1 \mid 9$ & &.$/ 911$ & 1491 & \\
\hline
\end{tabular}

منابع طبيعى مازندران - سارى، كارشناسان شركت سهامى نكان

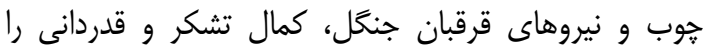
داريهم.
تشكر و قدردانى تامين

تامين بودجه اين طرح تحرديقاني توانى توسط اداره كل منابع

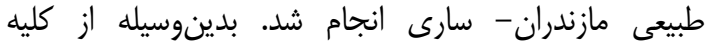

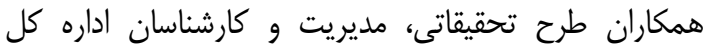


1. Alipour, A. and Sh. Mohammadnezhad Kiasari. 2017. Strategies for sustainable management of Iranian northern forests. Naghoos, Iran, $301 \mathrm{pp}$ (In Persian).

2. Babaeie-Kafaky, S., A. Mataji and N. Ahmadi Sani. 2009. Ecological capability assessment for multiple-use in forest area using Gis-based multiple criteria decision making approach. American Journal of Environmental Sciences, 5(6): 714-721.

3. Barbercheck, M.E., D.A. Neher, O. Anas, S.M. El-Allaf and T.R. Weicht. 2009. Response of soil invertebrates to disturbance across three resource regions in North Carolina. Environmental Monitoring and Assessment, 152(1-4): 283-98.

4. Bradford, M.A., G.M. Tordof, T. Egger, H. Jones and J.E. Newington. 2002. Microbiota, fauna, and mesh size interaction in litter decomposition.Oikos, 99(2): 317-323.

5. Callaham, M.A., D.D. Richter, D.C. Coleman and M. Hofmockel. 2006. Long term landuse effects on soil invertebrate communities in southern piedmont soils, USA. European Journal of Soil Biology, 42 (2): $150-156$.

6. Cusack, D. and F. Montagnini. 2004. The role of native species plantations in recovery of understory woody diversity in degraded pasturelands of Costa Rica. Forest Ecology and management, 188(5):115.

7. Duncan, R.S. and C.A. Chapman. 2003. Consequences of plantation harvest during tropical forest restoration in Uganda. Forest Ecology and Management, 173(8): 235-250.

8. Eslami, A., F. Jafari and M. Hasani. 2014. Relationship between qualitative and quantitative characteristics of regeneration with gap size in different types of Beech Stands (Case Study: Sourdar Anarestan Management Plan, Mazandaran). Iranian Forests Ecology, 2(3): 28-36.

9. Forest Management plan. 2012. District 2 Haftkhal, Series 4 Mozisa, Neka forests. Published by Natural Resources General Office of Sari, Forest and Rangelands Organization of Iran, 96 pp (In Persian)

10. Gorouyi, R., H. Yousefi and M. Alikhah-Asl. 2014. Ecological capability evaluation of Jiroft County for agricultural utilization, using GIS. Ecological Journal of Advanced Biological and Biomedical Research, 2(4): 1017-1022.

11. Haggar, J., K. Wightman and R. Fisher. 1997. The potential of plantations to foster woody regeneration within a deforested landscape in lowland Costa Rica. Forest Ecology and Management, 99 (2): 55-64.

12. Hashemi, S.A. 2018. Ecological capability evaluation for afforestation and forest expansion using geographic information system (GIS) in management area Caspian Sea. Anais da Academia Brasileria de Ciencias, 90 (4): 3761-3768.

13. Hassani, M. and M. Amani. 2005. The results of eight years of thinning on Maple (Acer velutinum) forestation (Case study: The Imamzadeh Abodulla of Amol). Iranian Journal of Forest and Poplar Research, 12(3): 339-370 (In Persian).

14. Hosseini, S.M., A. Muslem and Kh. Sageb-Talebi. 2002. Assessment of forest ecology power assessment methods. Journal of Environmental Studies, 26(25): 59-66. (In Persian)

15. Kiani, G., H. Jalilvand and R. Pourmajidian. 2013. Diameter increment of maple tree (Acer velutinum Boiss.) in plantations in Mazandaran (Case Study: Pahnekola Region). Iranian Forests Ecology, 1(2): 86-94 (In Persian).

16. Lavelle, P., T. Decaens, M. Aubert, S. Barot, M. Blouin, F. Bureau, P. Margerie, P. Mora and J.P. Rossi. 2006. Soil invertebrates and ecosystem services. European Journal of Soil Biology, 42(1): 3-15.

17. Marvie Mohajer, M.R. 2014. Silviculture. Fourth edition, University of Tehran Press, Iran, 387 pp (In Persian).

18. Mohammadnezhad Kiasari, Sh. 2017. Evaluation of the various methods of cultural operation (thinning) in the low land forestations (Hard woodand softwood) of Mazandaran province based on ecological capability (Case staudy: The forestations of Neka area). Final report of project, Mazandaran Agricultural and Natural Resources Research Center, Iran, 62 pp (In Persian).

19. Mohammadnezhad Kiasari, Sh. 2014. Final report of national research project: Investigation on adaptation of the world important hardwoods and softwoods in the north of Iran (Chamestan area: Pinaceae). Research Institute of Forests and Rangelands, Tehran, $73 \mathrm{pp}$.

20. Mohammadnezhad Kiasari, Sh., Sagheb-Talebi, K., Rahmani, R. and M. Akbarzadeh. 2013. Comparison plant diversity in natural forest and afforestations (Case study: Darabkola, mazandaran). Journal of Wood and Forest Science and Technology, 19(4): 59-76 (In Persian).

21. Mohammadnezhad Kiasari, Sh., Kh. Sagheb-Talebi, R. Rahmani and M. Amozad. 2013. Investigation on soil invertebrates' diversity at natural forests and refforestations of hardwood and softwood in sari area. Journal of Wood and Forest Science and Technology, 6(2): 55-69 (In Persian).

22. Mossadegh, A. 2015. Silviculture. Sixth Edition, University of Tehran Press, Tehran, Iran, 481p (In Persian).

23. Mulder, C. 2006. Driving forces from soil invertebrates to ecosystem functioning: the allometric perspective. Naturwissenschaften, 93(10): 1432-1904. 
24. Oatenand, D.K. and K.W. Larsen. 2008. Stand characteristics of three forest types within the dry interior forests of British Columbia, Canada: Implications for biodiversity. Forest Ecology and Management, 256(1-2): 114-120.

25. Rahmani, R. and H.Z. Mayvan. 2004. Diversity and assemblage structure of soil invertebrates in Beech, Hornbeam and Oak-Hornbeam forest types. Iranian Journal of Natural Research, 56(4): 425437 (In Persian).

26. Rouhi-Moghaddam, E., S.M. Hosseini, E. Ebrahimi, R. Rahmani and M. Tabari. 2007. The regeneration structure and biodiversity of trees and shrub species in understorey of pure plantations of oak and mixed with hornbeam in the Hyrcanian forests of Iran. Pakistan Journal of Biological Sciences, 10(8): 1281-1276.

27. Sagheb-Talebi, Kh., T. Sajedi and M. Pourhashemi. 2014. Forest of Iran. A Treasure from the Past, a Hope for the Future. Springer, $152 \mathrm{pp}$.

28. Seeber, J., G.U.H. Seeber, R. Langel, S. Scheu and E. Meyer. 2008. The effect of macro invertebrates and plant litter of different quality on the release of $\mathrm{N}$ from litter to plant on alpine pastureland. Biology and Fertility of Soil, 44(5): 783-790.

29. Siahipour, Z., B. Amanzadeh and Kh. Sagheb-Talebi. 2010. Preliminary results of impact of thinning on Norway spruce in Asalem region. Iranian Journal of Forest and Poplar Research, 18(1): 35-45.

30. Stankova, T.V. and U. Diéguez-Aranda. 2017. A two-component dynamic stand model of natural thinning. Forest Ecology and Management, 385: 264-280.

31. Sun, A., Y. Onda, K. Otsuki, H. Kato and T. gomi. 2017. The effect of script on forest floor evaporation in a Japanese cypress plantation. Agriculture and Forest Meteorology, 15: 48-57.

32. Varshosaz, K. and E. Mubarak Hassan. 2016. Harkaleh watershed ecological capability assessment for agriculture land with an emphasis on the sustainable development. European Journal of sustainable Development, 5(4): 121-128. 


\title{
Evaluation of the Two Methods for Thinning in Oak Plantation based on Ecological Capability (Case staudy: Neka Area, Mazadaran Province)
}

\author{
Shirzad Mohammadnezhad Kiasari ${ }^{1}$, Khosro Sagheb-Talebi ${ }^{2}$ and Kambiz Espahbodi ${ }^{3}$ \\ 1- Assistant Professor, Agriculture and Natural Resources Research and Education Center of Mazandaran, AREEO, \\ Mazandaran, I.R. Iran, (Corresponding author: ms.mohammadnezhadk@gmail.com) \\ 2- Associate Professor, Research Institute of Forests and Rangelands, AREEO, Tehran, I.R.Iran \\ 3- Associate Professor, Agriculture and Natural Resources Research and Education Center of Mazandaran, AREEO, \\ Mazandaran, I.R. Iran \\ Recived: January 3, $2018 \quad$ Accepted: December 4, 2018
}

\begin{abstract}
The study was aimed to assess of the Danish and Swiss methods of thinning in 20 years old plantations of Chestnut leaved Oak (Quercus castaneifolia C. A. Mey.) in terms of quantitative and qualitative characteristics of trees, natural regeneration, plant and soil invertebrate diversity. The study area is located in Neka forests, east of Mazandaran province in the Caspian region. This research was conducted under compeletly randomized block design (CRBD) with three replication and three treatments. Also in each sample plot, 3 microsample plot, each $49 \mathrm{~m}^{2}$ were randomly systematic selected. The quantitative and qualitative parameters of trees were measured in each sample plots by full callipering. In each microsample plot natural regeneration was counted. Also, whole plants were recognized in each microsample plot and their coverage percent were estimated. Moreover, in each microsample plot invertebrates were sampled, using core soil samples $\left(81 \mathrm{~cm}^{2}\right.$ cross section) to the depth of $10 \mathrm{~cm}$. The results of this research has been shown that the tree diameter at breast height, the abundance of quality tree and the number of natural regeneration in the thinned areas was more than the control area. Of course, the positive impact of the Danish method was greater than that of the Swiss method. Also this research has shown the control area created a more favorable situation due to the plant species diversity than the thinning areas. However the positive impact of the Danish method was greater than that of the Swiss method. On the other hand the Swiss thinning area created a more favorable situation due to the soil invertebrates' diversity than the Danish method. Overall this research emphasizes that the thinning operation with moderate intensity in Danish method is better than the Swiss method for forestations.
\end{abstract}

Keywords: Diversity, Forestation, Mazandaran, Thinning, Soil invertebrate diversity, Plant species 